\title{
Delayed Pharyngeal Extrusion of an Anterior Odontoid Screw
}

\author{
Eun Jung Lee', Jun Won Jang ${ }^{1}$, Seung Ho Choi ${ }^{2}$, Seung Chul Rhim ${ }^{1}$ \\ Departments of ${ }^{1}$ Neurological Surgery, of ${ }^{2}$ Otolaryngology, Asan Medical Center, University of Ulsan \\ College of Medicine, Seoul, Korea
}

\begin{abstract}
A 27-year-old woman with a type II odontoid fracture was treated by anterior odontoid screw fixation. Radiographic union at the fracture site was obtained 3months after surgery. Nearly 3 years after surgery, she presented at a local Ear, Nose, and Throat (ENT) clinic with a 2- month history of dysphagia. Laryngoscopy identified the head of the odontoid lag screw. Plain radiography showed that the head of the screw had migrated into the pharyngeal soft tissue. The atlantoaxial joint was stable, and computed tomography (CT) scans confirmed odontoid fracture fusion. The screw was found to be movable during endoscopy. The screw could be removed by using a transpharyngeal endoscopic approach under general anesthesia. The failure of the screw was considered to be due in part to malpositioning of the screw and in part to local infection. A transoropharyngeal endoscopic approach to remove the loose anterior odontoid screw was feasible.
\end{abstract}

Key Words: Odontoid process $\cdot$ Fracture fixation $\cdot$ Postoperative complications $\cdot$ Esophageal perforation

\section{INTRODUCTION}

Fractures of the axis involving the dens comprise 10-15\% of all cervical spine fractures and Anderson and D'Alonzo type II is the most common type of odontoid process fracture ${ }^{3)}$. Type II odontoid fractures have a high incidence of nonunion when immobilization alone is used: various groups have reported nonunion rates ranging from $5 \%$ to $60 \%{ }^{6,8}$.

Anterior screw fixation is increasingly being applied to selected patients with type II odontoid fractures and may now be the treatment of choice in this subpopulation. Direct screw fixation of odontoid fractures provides immediate stabilization, preserves normal atlantoaxial rotation, and allows for maximum cervical spine motion postoperatively. This technique results in a high fusion rates that range from $89 \%$ to $100 \%{ }^{1,5,8,12,14)}$ and obviates the need for postoperative halo immobilization or supplementary bone grafting. However, it is a technically challenging procedure, and problems such as malpositioning, fixation loss, and screw breakage can lead to serious complications

- Received: April 13, 2012 - Revised: September 4, 2012

- Accepted: September 26, 2012

Corresponding Author: Seung Chul Rhim, MD, PhD

Department of Neurological Surgery, Asan Medical Center, 388-1

Pungnap-2dong, Songpa-gu, Seoul 138-736, Korea

Tel: +82-2-3010-3554, Fax: +82-2-476-6738

Email:scrhim@amc.seoul.kr
The present report describes the case of a patient who underwent anterior screw placement for a type II odontoid fracture. Three years later she complained of dysphagia, and was diagnosed with dislodgement and pharyngeal extrusion of the anterior odontoid screw from the axis. The current clinical management of delayed screw loosening is discussed along with the possible reasons for why the technique failed.

\section{CASE REPORT}

A 27-year-old woman presented at the Accident and Emergency Department after a motor vehicle accident. She was reluctant to move her neck in any direction due to pain but was neurologically intact. She also complained multiple facial lacerations, epistaxis, and cerebrospinal fluid rhinorrhea. Plain radiography showed a fracture through the base of the odontoid
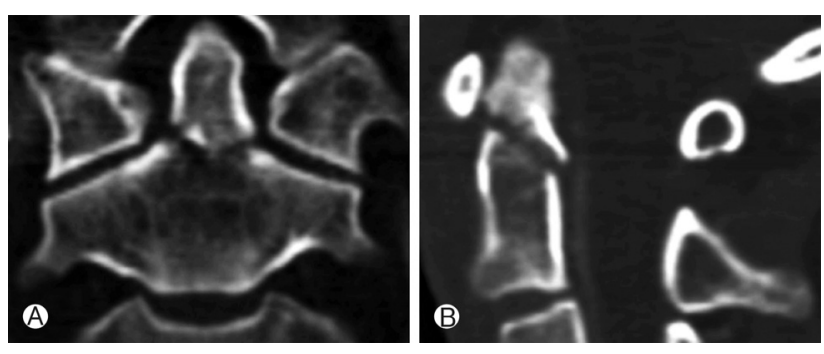

Fig. 1. Computed tomography (CT) scans of the cervical spine showing the type II odontoid fracture of the patient, which was typical for such fractures. (A) Coronal view, (B) Sagittal view. 
process (Fig. 1). Computed tomography (CT) scans confirmed the posterior oblique fracture but showed that the displacement was minimal. Magnetic resonance imaging showed an intact transverse ligament. The patient was also diagnosed with left frontal epidural hemorrhage and multiple facial bone fractures involving the frontal sinus, nasal bone, and left zygomaticomaxillary bone.

After undergoing plastic surgery for the multiple facial bone fractures, the patient underwent anterior odontoid fixation surgery conducted by the senior surgeon using a single $4.5 \times 40-\mathrm{mm}$ cannulated lag screw (Synthes PA, USA) 8 days post injury. Postoperatively she was neurologically intact and radiographic imaging showed good alignment of the odontoid process.

A week later, the patient complained of a sore throat and dysphagia. Erythematous neck swelling was visible on the surgical wound. A neck CT showed significant fluid collection with rim enhancement in the right lateral neck and retropharyngeal area at the level of C5-6 (Fig. 2A). Ultra-sonography-guided aspiration of pus was done twice. The pus was cultured and a methicillin resistant Staphylococcus aureus was isolated. The patient was placed on intravenous vancomycin $1 \mathrm{~g}$ two times a day for a month. Thereafter, she recovered completely, as indicated by clinical and radiographic examinations (Fig. 2B).

Radiographic imaging for anterior odontoid fixation was performed on discharge and 3, 6 and 12 months postoperatively: it consisted of plain radiographs of the cervical spine in the anteroposterior and lateral projections with flexion and extension views and fine-cut CT scans with sagittal reconstruction. Radiographic union at the fracture site was obtained 3 months after surgery. A trabeculation across the fracture site and absence of movement was demonstrated by flexionextension lateral radiographs. The screw placement was considered to be excellent. There was no evidence of instrumental failure until 12 months after surgery (Fig. 3A).

Nearly 3 years after surgery, the patient presented at a local ENT clinic with a 2-month history of dysphagia and the sense
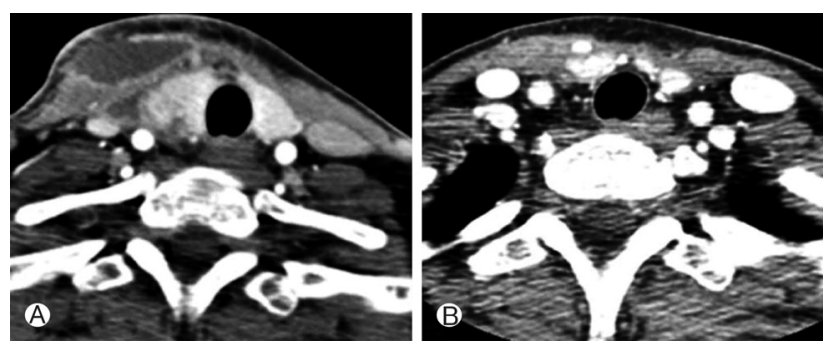

Fig. 2. Neck CT scans show significant fluid collection with rim enhancement in the right lateral neck and retropharyngeal area at the level of C5-6 (A). Follow-up CT scan a month after treatment shows no residual abscess (B). of a foreign body in the throat. These symptoms started after the patient had suffered from severe paroxysmal coughing due to influenza for a month. Laryngoscopy identified the head of the odontoid screw. Plain radiography showed that the head of the screw had migrated into the pharyngeal soft tissue (Fig. 3B). Flexion and extension radiographs did not detect motion at the fracture and indicated maintenance of the postoperative alignment. CT scans confirmed fusion of the odontoid fracture.

The patient was prepared for possible combined spine and otolaryngology intervention. The plan was to first attempt screw removal using forceps during suspension laryngoscopy, and if that failed, a neurosurgical anterior approach would be undertaken. Fiber-optic endotracheal intubation was performed while the patient was conscious. After induction of general anesthesia, a suspension laryngoscopy showed the screw head was surrounded by granulation tissue in the vallecular fossa. The screw was found to be movable and was easily removed by using small forceps under laryngomicroscopy (Fig. 4). A suture was not necessary. Flexion and extension
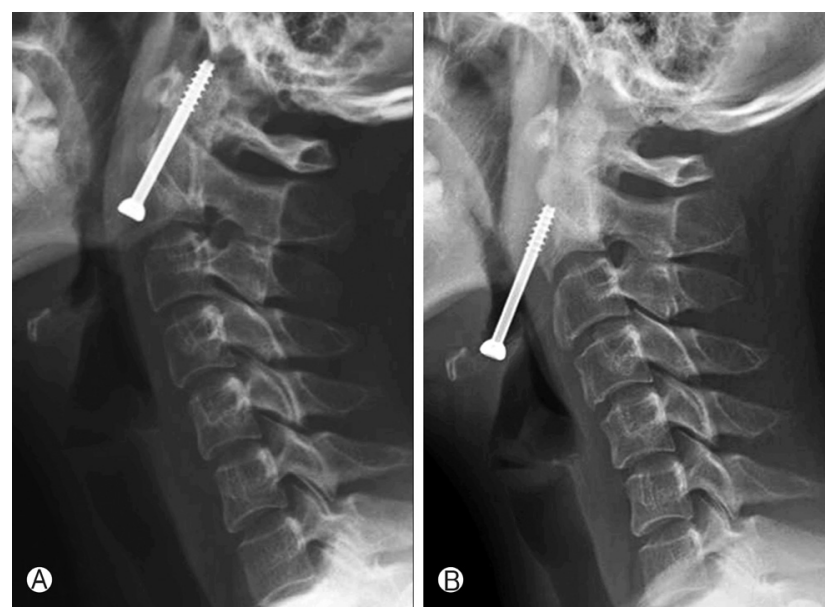

Fig. 3. Plain $X$-ray lateral images of the cervical spine obtained 3 months $(A)$ and 3 years $(B)$ postoperatively.

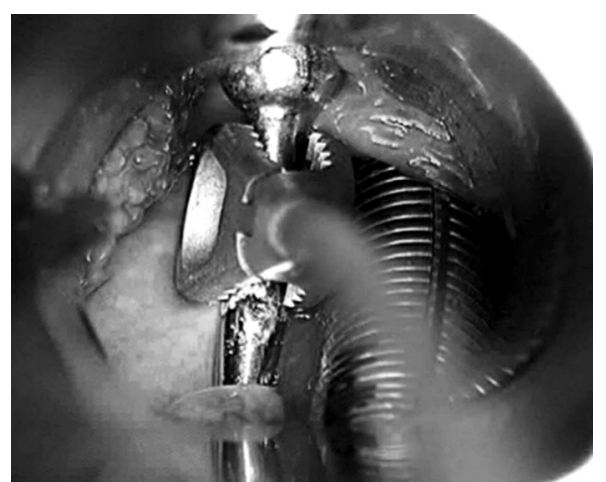

Fig. 4. The extruded anterior odontoid screw was removed under suspension laryngoscopy. 
films of the cervical spine showed no abnormal movement. The dysphagia and neck pain resolved shortly after the removal of the screw, and the patient was discharged the day after surgery.

\section{DISCUSSION}

A variety of complications associated with anterior odontoid fixation appear to relate to the instrumentation. In a review of several series of anterior odontoid fixation, Subach et al. ${ }^{14)}$ detected a combined screw malposition rate of $3.2 \%$ (8/252 patients), a screw pullout rate of $3.2 \%$, and a screw fracture rate of $0.3 \%$. However, all reported dislodgements of the screws occurred immediately after surgery or before bony fusion was achieved, and they were not accompanied with pharyngeal perforation ${ }^{2,4,7,12)}$. However, several reports describe the delayed esophageal or pharyngeal perforation that is due to anterior cervical spine fusion (rather than anterior odontoid fixation) ${ }^{9,11,13)}$. In these cases, a major factor contributing to the screw failure was the malpositioning of the screws in the vertebral body, and their inadequate engagement in the plate.

In our case, postoperative CT scans showed that the screw purchase was secure in the apical cortical bone of the dens fragment (Fig. 5A). However, the entry point was at a point $4 \mathrm{~mm}$ above the anterior-inferior margin of the $\mathrm{C} 2$ body and the screw was inserted into the ventral C2 body, which made the screw relatively long for a lag screw (Fig. 5A). This techni-
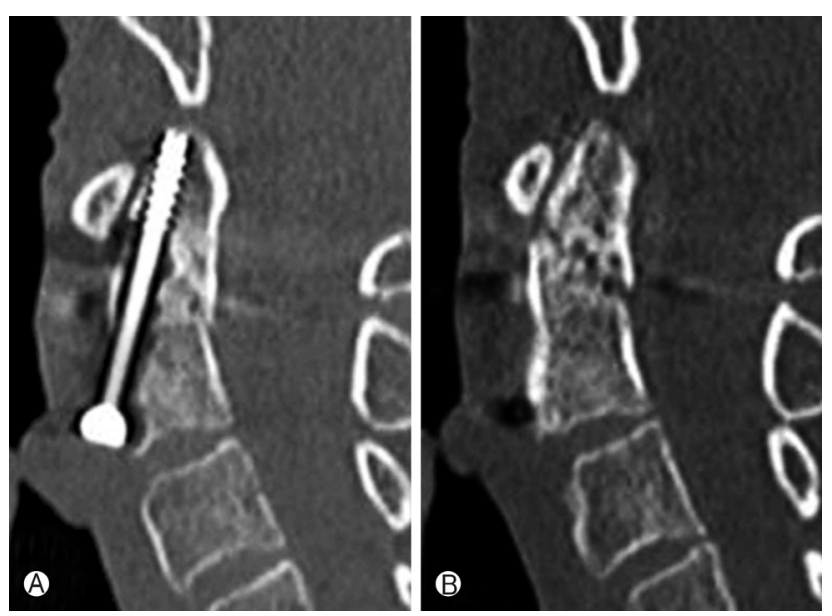

Fig. 5. Postoperative CT scans 3 months after the operation show that the bicortical purchase of the screw is obtained $(A)$, however, the entry point is at $4 \mathrm{~mm}$ above the anterior-inferior margin of the C2 body, which made the screw relatively long for a lag screw and the interfragmentary compression is not achieved (B). Pathological resorption of the bone around the screw is noted, while osseous union of odontoid fracture begins to appear (A). cal error was considered to be due to intraoperative insufficient cervical extension. The alignment of the fracture fragment appeared to be satisfactory but the interfragmentary compression was not achieved by the lag screw (Fig. 5B). Furthermore, the patient suffered from a retropharyngeal abscess postoperatively. Although the retropharyngeal abscess was at the level of C5, 6 and was cured completely, the infection may have spread to the $\mathrm{C} 2$ vertebra. Retrospective cervical CT revealed pathological resorption of the bone around the screw, which began to appear 3 months after surgery, while osseous union was also noted (Fig. 5A). Normal repetitive motion would have eventually loosened the screw over time after successful fusion. Notably, persistent paroxysmal coughing ( $>1$ month) and repetitive friction of a redundant odontoid screw has been suggested to lead to pressure necrosis in the posterior pharyngeal wall and dislodgement of the screw ${ }^{10)}$.

The esophagus and pharynx are at risk of perforation due to the close anatomical relationship between the esophagus, pharynx and anterior cervical construct. Esophageal perforation complication can be fatal and can demand interdisciplinary treatment ${ }^{15)}$. In the present case, endoscopic transpharyngeal removal was performed without pharyngeal closure, and the symptoms resolved. Hence, a transpharyngeal endoscopic approach to remove a loose anterior odontoid screw was found to be feasible.

\section{CONCLUSION}

The present report appears to be the first to describe the delayed pharyngeal extrusion of an anterior odontoid screw after achievement of union. The possible reasons for screw failure were reviewed. An exact entry point, an adequate trajectory, and bicortical purchase of the screw are all necessary for anterior odontoid fixation. We recommend that the first approach to remove a loose anterior odontoid screw should be a transoropharyngeal endoscopic approach.

\section{REFERENCES}

1. Isolated fractures of the axis in adults. Neurosurgery 50:S125139, 2002

2. Aebi M, Etter C, Coscia M: Fractures of the odontoid process. Treatment with anterior screw fixation. Spine (Phila Pa 1976) 14:1065-1070, 1989

3. Anderson LD, D'Alonzo RT: Fractures of the odontoid process of the axis. J Bone Joint Surg Am 56:1663-1674, 1974

4. Bohler J: Anterior stabilization for acute fractures and non-unions of the dens. J Bone Joint Surg Am 64:18-27, 1982

5. Borne GM, Bedou GL, Pinaudeau M, Cristino G, Hussein A: Odontoid process fracture osteosynthesis with a direct screw 
fixation technique in nine consecutive cases. J Neurosurg 68: 223-226, 1988

6. Chiba K, Fujimura Y, Toyama Y, Fujii E, Nakanishi T, Hirabayashi K: Treatment protocol for fractures of the odontoid process. J Spinal Disord 9:267-276, 1996

7. Clark CR, White AA $3^{\text {rd: }}$ Fractures of the dens. A multicenter study. J Bone Joint Surg Am 67:1340-1348, 1985

8. Geisler FH, Cheng C, Poka A, Brumback RJ: Anterior screw fixation of posteriorly displaced type II odontoid fractures. Neurosurgery 25:30-37, 1989

9. Geyer TE, Foy MA: Oral extrusion of a screw after anterior cervical spine plating. Spine (Phila Pa 1976) 26:1814-1816, 2001

10. Hanci M, Toprak M, Sarioglu AC, Kaynar MY, Uzan M, Islak C: Oesophageal perforation subsequent to anterior cervical spine screw/plate fixation. Paraplegia 33:606-609, 1995
11. Kim SB, Oh SH, Kim YS, Ko Y, Chung WS: Delayed esophageal perforation after an anterior cervical plating. Korean J Spine 1: 375-378, 2004

12. Montesano PX, Anderson PA, Schlehr F, Thalgott JS, Lowrey $\mathrm{G}$ : Odontoid fractures treated by anterior odontoid screw fixation. Spine (Phila Pa 1976) 16:S33-S37, 1991

13. Nourbakhsh A, Garges KJ: Esophageal perforation with a locking screw: a case report and review of the literature. Spine (Phila Pa 1976) 32:E428-435, 2007

14. Subach BR, Morone MA, Haid RW Jr, McLaughlin MR, Rodts GR, Comey CH: Management of acute odontoid fractures with single-screw anterior fixation. Neurosurgery 45:812-819, 1999

15. von Rahden BH, Stein HJ, Scherer MA: Late hypopharyngoesophageal perforation after cervical spine surgery: proposal of a therapeutic strategy. Eur Spine J 14:880-886, 2005 